\title{
VARIATIONAL METHOD FOR ADIABATIC COMPRESSION OF PLASMA WITH POLOIDAL AND TOROIDAL ROTATION
}

\author{
L. J. PaN \\ Institute of Mechanics, Chinese Academy of Sciences, Beijing, China and Department of Physics, \\ Imperial College, London SW7 2BZ, U.K. \\ (Received 6 April 1987; and in revised form 12 January 1989)
}

\begin{abstract}
A variational method is developed for adiabatic compression of plasma with both poloidal and toroidal rotation.
\end{abstract}

\section{INTRODUCTION}

ADIABATIC COMPRESSION HEATING is considered as one of the methods to further increase the temperature of the confined plasma for a number of experiments such as the Russian T-14 Tokamak, the German ZEPHYR, and the PDX and spheromak plasma at Los Alamos. It is known that the large toroidally unbalanced forces associated with neutral beam injection induce plasma flows which may approach a modest fraction of the hydrogen ion thermal velocity (BraU et al., 1983). Therefore considerable interest in the behaviour of a confined plasma with mass flow has grown in recent years. The presence of large flows may cause an appreciable change in the equilibrium state as well as in its stability.

It is essential to develop a computationally fast, yet sufficiently accurate, method that will enable a thorough investigation of an adiabatically compressed plasma with poloidal and toroidal rotation. The adiabatic compression of a plasma with poloidal rotation has not yet, to our knowledge, been treated analytically before. The work in this paper aims to develop a variational method for the computation of the adiabatic compression of a plasma with poloidal and toroidal rotation in a realistic geometry.

For the work described here, transport processes are neglected; therefore the plasma is governed by ideal MHD equations. In reality, the final plasma states may be strongly affected by transport processes, so a full calculation with transport is also required in addition to the present work. A work on this line is reported (KERNER and WeITZER, 1985). Another basic idea in the adiabatic compression approximation is to assume that the compression takes place on a time scale much slower than a typical Alfvén wave transit time across the major dimensions of the device, yet fast enough for transport to be neglected. The wave causes a fast equilibrium, hence the plasma may be seen as creeping from one equilibrium state to the next, and a sequence of different equilibrium states may be determined without following the full dynamics. GRAD et al. (1975) treated the adiabatic compression of plasma without flows by introducing a so-called "queer differential equation" to describe the equilibrium states and the alternating dimensions method to solve the equation. For the case of plasmas with toroidal rotation, HAMEIRI (1983a) introduced a variational principle due to Woltijer to determine the free functions involved in the Grad-Shafranov equation, so 
that the alternating dimensions method can still be applied. This technique can significantly increase the computer time and storage requirements. We notice that the efficient variational method developed by LAO et al. (1981) and WU and PAN (1983) independently for finding approximate solutions to the Grad-Shafranov can be extended to the generalized Grad-Shafranov equation for our case. In LAO's scheme, the flux surface coordinates $(R(\eta, \theta), Z(\eta, \theta))$ are expanded in Fourier series in a poloidal angle $\theta$, where $\eta$ labels the magnetic surface. Through the use of the variational method, the Fourier amplitudes of $R$ and $Z$ are obtained by solving a set of coupled ordinary differential equations, which are the moment of the Grad-Shafranov equation.

This technique is further developed in this paper by constructing a new Lagrangian appropriate to the generalized Grad-Shafranov equation. There is some difficulty in determining the arbitrary functions involved in our equation. However, these can be approximately expressed in terms of the given constants of plasma motion and the geometric factor of flux surface, if beta or the Mach number is small. Consequently this complicated problem is greatly simplified to solving a set of nonlinear coupled ordinary differential equations.

\section{THE GENERALIZED GRAD-SHAFRANOV EQUATION AND CONSERVATION-LAW CONSTRAINT}

For simplicity, the generalized Grad-Shafranov equation obtained by HAMEIRI (1983b) for an axisymmetric ideal MHD equilibrium state with flow is shown below without repeating the derivation:

$$
\nabla \cdot\left[\left(1-\frac{F^{2}}{\rho}\right) \frac{\nabla \psi}{R^{2}}\right]+\bar{U} \cdot \bar{B} \dot{F}+R \rho U_{\phi} \dot{\Omega}+\frac{1}{R} B_{\phi} \dot{I}-\frac{1}{\gamma-1} \rho^{\gamma} \dot{S}+\rho \dot{H}=0
$$

where the symbols $\bar{B}, \psi, \bar{u}, P, \rho, s$ and $\gamma$ have their usual meaning and the total magnetic field $\bar{B}$ is defined in toroidal coordinates as

$$
\bar{B}=\frac{1}{R} \nabla \psi \times \nabla \phi+B_{\phi} \hat{\phi}
$$

where $\phi$ is the ignorable toroidal angle. The dot in equation (1) denotes $d / d \psi$, and $F(\psi), \Omega(\psi), H(\psi)$ and $S(\psi)$ are five arbitrary functions of $\psi$, which have the following relations derived from the ideal MHD equations (HAMEIRI, 1983b) :

$$
\begin{gathered}
\bar{U}=\rho^{-1} F \bar{B}+R \Omega \hat{\phi} \\
B_{\phi}=\frac{1}{1-F^{2} / \rho}\left[\frac{I}{R}+R F \Omega\right] \\
\frac{F^{2}}{2 \rho^{2}}\left[\frac{1}{R^{2}}(\nabla \psi)^{2}+B_{\phi}^{2}\right]-\frac{1}{2} R^{2} \Omega^{2}+\frac{\gamma}{\gamma-1} S \rho^{\gamma-1}=H(\psi) .
\end{gathered}
$$


Equation (3) shows that the lines of force are frozen into the fluid; hence any closed constant $\psi$ surface always consists of the same fluid particles, and the flow has one component parallel to $\bar{B}$ with magnitude proportional to $F(\psi)$, superimposed on a rigid rotation of each individual flux surface. The familiar function $I(\psi)$ is not exactly the usual total poloidal current, and should be modified by the plasma flow as shown in equation (4). $H(\psi)$ is the analogue of the Bernoulli function in fluid dynamics. In deriving the above relations, the equation of state $P=S(\psi) \rho^{\gamma}$ was used.

Equation (1) is an elliptic PDE (HAMEIRI, 1983a), when

$$
F^{2} / \rho<\gamma P /\left(\gamma P+\bar{B}^{2}\right) .
$$

Therefore it requires either Dirichlet or Neumann boundary conditions to solve the problem of slow poroidal flow.

What distinguishes the present problem from the usual one in a static equilibrium state is that, during the adiabatic compression, only $S(\psi)$ remains unchanged, the other functions $F(\psi), \Omega(\psi), H(\psi)$ and $I(\psi)$, instead of being given, must be determined in such a way that the dynamical conservation laws of an evolving plasma are obeyed. It can be shown that these conservation-law constraints are

mass

$$
\langle\rho\rangle=m(\psi) \psi^{\prime}
$$

angular momentum

$$
\left\langle R \rho U_{\phi}\right\rangle=l(\psi) \psi^{\prime}
$$

toroidal flux

$$
\left\langle B_{\phi} / R\right\rangle=f(\psi) \psi^{\prime}
$$

circulation

$$
\langle\bar{U} \cdot \bar{B}\rangle=C(\psi) \psi^{\prime}
$$

where

$$
\langle A\rangle=\oint A \mathrm{~d} \Sigma /|\nabla v|=\frac{\mathrm{d}}{\mathrm{d} v} \int_{\psi} A \mathrm{~d} v
$$

with

$$
\oint \mathrm{d} \Sigma /|\nabla v|=1 \quad \text { and }()^{\prime}=\frac{\mathrm{d}}{\mathrm{d} v}
$$

where $\Sigma$ is the area of flux surface and $v$ is the volume inside a closed flux surface. The volume integral in (10) is taken within each flux tube. $\mathrm{d} v$ is the volume element in a plasma. Equations (6)-(9) show that within each moving flux tube the mass angular momentum, toroidal magnetic flux and circulation remain constant during compression. 


\section{VARIATIONAL METHOD}

Consider the volume integral

$$
Q=\int_{v} L\left(\psi, \psi_{R}, \psi_{Z}, R\right) \mathrm{d} R \mathrm{~d} Z
$$

where $L$ is the Lagrangian and the integration is over the entire plasma volume, $V$. A variation of $Q$ with respect to $\psi$, subject to the boundary condition $\delta \psi=0$, yields the Euler equation

$$
\frac{\partial L}{\partial \psi}-\frac{\partial}{\partial R} \frac{\partial L}{\partial \psi_{R}}-\frac{\partial}{\partial Z} \frac{\partial L}{\partial \psi_{Z}}=0
$$

Since the Lagrangian (LAU et al, 1981; WU and PAN, 1983), whose variation gives the Grad-Shafranov equation, is

$$
L=\left(\frac{1}{2} B_{\mathrm{P}}^{2}-P-\frac{1}{2} B_{\phi}^{2}\right) R
$$

we may write the Lagrangian for equation (1) as

$$
L=\left[\frac{1}{2} B_{\mathrm{P}}^{2}-P-\frac{1}{2} B_{\phi}^{2}-\rho U_{\mathrm{P}}^{2}\right] R
$$

or

$$
L=\left[\frac{1}{2 R^{2}}(\nabla \psi)^{2}\left(1-\frac{F^{2}}{\rho}\right)-\frac{1 / 2}{1-F^{2} / \rho}\left(\frac{I}{R}+R F \Omega\right)^{2}-\frac{\rho R^{2} \Omega^{2}}{2}+\frac{\rho^{\gamma} S}{\gamma-1}-\rho H\right] R
$$

It can be shown that $\partial L / \partial \rho=0$.

Substitution of (14) in (13) and some calculations lead to the generalized GradShafranov equation (1). It shows that the Lagrangian $L$ given in (14) is the correct Lagrangian associated with (1), and that $Q$ is stationary with respect to $\psi$ for $\psi$ satisfying the generalized Grad-Shafranov equation. Since the Lagrangian has been found, the variational method developed by LAO et al. (1981) for the usual GradShafranov equation can be extended to our case. Notice that $\rho=\rho(\psi, R, \nabla \psi)$; however, in the above calculations $\rho$ may be taken as a constant because of the property $\partial L / \partial \rho=0$.

\subsection{Transformed Euler equation}

Following LAO et al. (1981), we consider the transformation in equation (12) of the independent variables $(R, Z)$ to $(\eta, \theta)$ coordinates

$$
Q=\int_{0}^{a} \int_{0}^{2 \pi} L^{*}\left(R, R_{\eta}, R_{\theta}, Z_{\eta}, Z_{\theta}, \psi, \psi_{\eta}\right) \mathrm{d} \eta \mathrm{d} \theta
$$


where $\eta=a$ denotes the outermost flux surface and the subscripts $\eta$ and $\theta$ denote differentiation with respect to these variables. The transformed Lagrangian is given by

$$
\begin{aligned}
L^{*}=\left(1-F^{2} / \rho\right)\left(R_{\theta}^{2}+Z_{\theta}^{2}\right) & \frac{\psi^{\prime 2}}{2 R \tau} \\
& -R \tau\left[\frac{1}{1-F^{2} / \rho}\left(\frac{I^{2}}{2 R^{2}}+I F R+\frac{\rho R^{2} \Omega^{2}}{2}\right)-\frac{\rho^{y}}{\gamma-1} S+\rho H\right]
\end{aligned}
$$

with

$$
\tau=R_{\theta} Z_{\eta}-R_{\eta} Z_{\theta} \quad \text { and } \quad()^{\prime}=\frac{\mathrm{d}}{\mathrm{d} \eta} .
$$

In the representation of $Q$ given by (15), $R$ and $Z$ are taken as dependent variables. Since $Q$ is a scalar value, its stationary value must be independent of any transformation. Therefore the variation of $Q$ with respect to $R$ and $Z$ subject to the boundary constraints $\delta R=\delta Z=0$ should reproduce the inverse equilibrium equation (1) as shown below.

The variations of $Q$ with respect to $R$ and $Z$ with the fixed boundary constraints $\delta R=0$ and $\delta Z=0$ yield the Euler equations, respectively

$$
\frac{\partial L^{*}}{\partial R}-\frac{\partial}{\partial \eta} \frac{\partial L^{*}}{\partial R_{\eta}}-\frac{\partial}{\partial \theta} \frac{\partial L^{*}}{\partial R_{\theta}}=-\psi^{\prime} Z_{\theta} G=0
$$

and

$$
-\frac{\partial}{\partial \eta} \frac{\partial L^{*}}{\partial Z_{\eta}}-\frac{\partial}{\partial \theta} \frac{\partial L^{*}}{\partial Z_{\theta}}=\psi^{\prime} R_{\theta} G=0
$$

where

$$
\begin{aligned}
G=\frac{1}{\tau}\left\{\frac{\partial}{\partial \eta}\left[\left(1-\frac{F^{2}}{\rho}\right) \frac{\psi^{\prime}\left(R_{\theta}^{2}+Z_{\theta}^{2}\right)}{\tau R}\right]\right. & -\frac{\partial}{\partial \theta}\left[\left(1-\frac{F^{2}}{\rho}\right) \frac{\psi^{\prime}}{R \tau}\left(R_{\eta} Z_{\theta}+Z_{\eta} R_{\theta}\right)\right] \\
+R & {\left.\left[\tilde{U} \cdot \bar{B} \dot{F}+R \rho u_{\theta} \dot{\Omega}+B_{\theta} R \dot{I}+\rho \dot{H}-\frac{\rho^{\gamma} \dot{S}}{\gamma-1}\right]\right\} . }
\end{aligned}
$$

It can easily be verified that $G=0$ is the inverse generalized Grad-Shafranov equation (1).

\subsection{Fourier series transformation of coordinates}

Following LAO et al. (1981), the coordinate transformation as a Fourier series in $\theta$ 


$$
R(\eta, \theta)=\sum_{n=0}^{\infty} R_{n}(\eta) \cos n \theta
$$

and

$$
Z(\eta, \theta)=\sum_{n=1}^{\infty} Z_{n}(\eta) \sin n \theta
$$

is adopted, where $\eta(\psi)$ is the flux surface label, $\theta$ is a poloidal angle increasing $2 \pi$ the short way around the torus, and $\psi$ is defined as the magnetic flux enclosed between the flux surface $\eta(\psi)$ and the axis of symmetry. The amplitudes $R_{0}(\eta), R_{1}(\eta)$ and $Z_{1}(\eta)$ describe the shift, the minor radius and the ellipticity of the flux surface, respectively, whereas the amplitudes $R_{2}(\eta)$ and $Z_{2}(\eta)$ describe the triangularity of the flux surface. It is found that, in practice, only a few leading terms are sufficient to describe the flux contours. Thus, the present problem may be reduced to a problem for solving just a few coupled nonlinear ordinary differential equations for $R_{n}$ and $Z_{n}$.

\subsection{Moment equation for the inverse mapping}

It has been proven that the flux surface described either by $\psi(R, Z)$, or by $R(\eta, \theta)$ and $Z(\eta, \theta)$, is such that $Q$ is stationary. This provides a method to calculate the Fourier amplitudes $R_{n}$ and $Z_{n}$ in such a way that $Q$ is stationary with respect to the variations of these amplitudes subject to the boundary conditions $\delta R_{n}=\delta Z_{n}$.

The use of the specific convenient truncated Fourier representation (LAO et al., 1981)

$$
\begin{aligned}
& R(\eta, \theta)=R_{0}(\eta)-R_{1}(\eta) \cos \theta+\sum_{n=2}^{n_{T}} R_{n}(\eta) \cos n \theta \\
& Z(\eta, \theta)=E(\eta)\left[R_{1}(\eta) \sin \theta+\sum_{n=2}^{n_{T}} R_{n}(\eta) \sin n \theta\right]
\end{aligned}
$$

and the variations of $Q$ with respect to $R_{n}(\eta)$ and $E(\eta)$ lead to a new set of Euler equations

$$
\begin{gathered}
\left\langle\left\langle\frac{\partial L^{*}}{\partial R_{n}}-\frac{\partial}{\partial \eta} \frac{\partial L^{*}}{\partial R_{n}^{\prime}}\right\rangle\right\rangle=\left\langle\left\langle M_{n} G\right\rangle\right\rangle=0 ; n=0,1, \ldots, n_{T} \\
\left\langle\left\langle\frac{\partial L^{*}}{\partial E}-\frac{\partial}{\partial \eta} \frac{\partial L^{*}}{\partial E^{\prime}}\right\rangle\right)=\left\langle\left\langle M_{E} G\right\rangle\right\rangle=0 ;
\end{gathered}
$$

where the prime denotes differentiation with respect to $\eta$, and $\langle\langle f\rangle\rangle$ is the poloidal angle averaging operator defined as 


$$
\langle\langle f\rangle\rangle=\int_{0}^{2 \pi} f \mathrm{~d} \theta / 2 \pi
$$

and

$$
\begin{gathered}
M_{0}=-Z_{\theta} \\
M_{1}=E R_{\theta} \sin \theta+Z_{\theta} \cos \theta \\
M_{2}=E R_{\theta} \sin 2 \theta-Z_{\theta} \cos 2 \theta \\
M_{n}=E R_{\theta} \sin n \theta-Z_{\theta} \cos n \theta ; \quad n=3, \ldots, n_{T} \\
M_{E}=\sum_{n=1}^{n_{T}} R_{n} R_{\theta} \sin n \theta
\end{gathered}
$$

Equations (25) and (26) comprise a system of coupled, second order, nonlinear ordinary differential equations in $\eta$ for the Fourier amplitudes $E$ and $R_{n}$. The five free functions involved in $G$ will be found from the four conservation-law constraints (6)(9) and the Bernoulii equation (5). Here a truncated Fourier series is taken, because the flux surface can be approximately described by a few leading terms.

\section{DETERMINATION OF $I(\psi), H(\psi), \Omega(\psi), F(\psi)$ AND $\rho(\psi, R, \nabla \psi)$}

The poloidal and toroidal rotation velocities reported by BRAU et al. (1983) are less than $3 \times 10^{5} \mathrm{~cm} \mathrm{~s}^{-1}$, thus we assume the plasma velocity given by (3) is small compared with the Alfvén or sound speed

$$
\frac{R \Omega}{\sqrt{\gamma P / \rho}}=0(\varepsilon)
$$

and parallel Mach number

$$
M_{\mid}=u_{\varphi} /\left(\frac{\gamma P}{\rho}\right)^{1 / 2}=0(\varepsilon),
$$

or

$$
\frac{F}{\sqrt{\rho}}=\frac{U_{\mathrm{l}}}{V_{\mathrm{A}}}=\sqrt{\frac{\gamma}{2}} M_{\mathrm{i}} \sqrt{\beta}=0(\varepsilon)<\left(\gamma P /\left(\gamma P+B^{2}\right)\right)^{1 / 2},
$$

where

$$
V_{\mathrm{A}}=B / \sqrt{\rho}, \quad U_{\|}=F B / \rho, \quad \beta=P /\left(\frac{B^{2}}{2}\right) .
$$


Here $U_{\|}$denotes the plasma velocity parallel to the magnetic field up to a rigid rotation of each flux surface. In this case equation (1) is an elliptic PDE.

With the assumption given in equation (34) in mind, from (3) and (5), we find the terms $F^{2} / \rho, R \bar{U} \cdot \bar{B} \dot{F}, R^{2} \rho U_{\theta} \dot{\Omega}$ in $G$ shown in equation (20) are of order $\varepsilon^{2}$ in comparison with the rest of the terms. Therefore, for an accuracy of order $\varepsilon^{2}$, we calculate $F, \Omega$ to order $\varepsilon$ and $H, I, P$ to order $\varepsilon^{2}$ by using equations (3)-(9) as shown below:

$$
\begin{aligned}
& \rho=\rho^{(0)}+\rho^{(2)} \\
& I=I^{(0)}+I^{(2)} \\
& H=H^{(0)}+H^{(2)} \\
& F=F^{(1)} \\
& \Omega=\Omega^{(1)} \\
& \rho^{(0)}=m \psi^{\prime} \\
& I^{(0)}=f \psi^{\prime} /\left\langle\frac{1}{R^{2}}\right\rangle \\
& H^{(0)}=\frac{\gamma}{\gamma-1} S \rho^{(0) \gamma-1}=\frac{\gamma}{\gamma-1} S\left(m \psi^{\prime}\right)^{\gamma-1} \\
& F^{(1)}=\left(l f /\left\langle\frac{1}{R^{2}}\right\rangle-m\left\langle R^{2}\right\rangle\right) /\left[f^{2} /\left\langle\frac{1}{R^{2}}\right\rangle^{2}-\left\langle R^{2}\right\rangle\left(\left\langle\frac{(\nabla \psi)^{2}}{R^{2}}\right\rangle / \psi^{\prime 2}+\frac{f^{2}}{\left\langle\frac{1}{R^{2}}\right\rangle}\right)\right] \\
& \Omega^{(1)}=\frac{l}{m\left\langle R^{2}\right\rangle}+\left(\frac{f}{\left\langle\frac{1}{R^{2}}\right\rangle}-\frac{l f^{2}}{\left\langle R^{2}\right\rangle\left\langle\frac{1}{R^{2}}\right\rangle^{2} m}\right) / \\
& {\left[f^{2} /\left\langle\frac{1}{R^{2}}\right\rangle^{2}-\left\langle R^{2}\right\rangle\left(\left\langle\frac{(\nabla \psi)^{2}}{R^{2}}\right\rangle / \psi^{\prime 2}+\frac{f^{2}}{\left\langle\frac{1}{R^{2}}\right\rangle}\right)\right]} \\
& I^{(2)}=-F^{(1)}\left(\Omega^{(1)}+\frac{F^{(1)} f}{m}\right) /\left\langle\frac{1}{R^{2}}\right\rangle
\end{aligned}
$$




$$
\begin{gathered}
H^{(2)}=\frac{F^{(1) 2}}{2\left(m \psi^{\prime}\right)^{2}}\left(\left\langle\frac{1}{R^{2}}(\nabla \psi)^{2}\right\rangle+\frac{f^{2} \psi^{\prime 2}}{\left\langle\frac{1}{R^{2}}\right\rangle}-\frac{1}{2}\left\langle R^{2}\right\rangle \Omega^{(1) 2}\right) \\
\rho^{(2)}=\left[H^{(2)}-\frac{F^{(1) 2}}{2 m^{2} \psi^{\prime 2}}\left(\frac{1}{R^{2}}(\nabla \psi)^{2}+\frac{I^{(0) 2}}{R^{2}}\right)+\frac{1}{2} \Omega_{0}^{(1) 2} R^{2}\right] \frac{\left(m \psi^{\prime}\right)^{2-\gamma}}{\gamma S}
\end{gathered}
$$

where the superscript (2) denotes order $\varepsilon^{2}$.

Since all free functions including $P$ are explicitly expressed in terms of the unchanged conservative quantities and geometric factors as shown in equations (37)-(49), thereafter the set of coupled ordinary differential equations (25) and (26) can be solved numerically by substituting (42)-(49) into $G$, with different boundary conditions prescribed by the compression method.

It is interesting to investigate whether the plasma can speed up to a supersonic level due to the conservation of angular momentum during a compression. At this point, one can calculate the free functions and $\rho$ to higher orders of the Mach number in a similar way to that just developed. In reality, the poloidal velocity damps out much faster than the toroidal velocity due to the fast dissipation of parallel stress, and therefore the compression of rotating plasmas without poloidal flow is relevant (PAN and FU, 1989) for the case when the compression is much slower than the parallel thermalization time.

To conclude, the variational method developed in this paper has provided a computationally efficient and powerful method for an adiabatic compression of a plasma with both toroidal and poloidal rotation. The essential idea of the variational method was applied successfully by Wu and PAN (1983) elsewhere.

A numerical computation concerning the present problem will be reported later. This technique has applications to both laboratory and astrophysical plasmas. The compression or equilibrium state of plasmas with larger poloidal flows is not discussed here because equation (1) becomes hyperbolic in this case.

Acknowledgement-The author is very much indebted to Professor M. G. HAINes for his invitation to Imperial College and for useful discussions and assistance in the publication of this paper.

\section{REFERENCES}

Brau K., Bitter M., Goldston R. J., Manos D, McGuire K. and Slckewer S. (1983) Nucl. Fusion 23, No. 12.

Grad H., Hu P. N. and Stevens D. C. (1975) Proc. natn. Acad. Sci. U.S.A. 72, 3789.

Hameiri E. (1983a) Phys. Rev. A27, 1259.

Hameiri E. (1983b) Physics Fluids 26, 230.

KERNER W. and WEITZNER H. (1985) Courant Institute of Mathematical Sciences, Magneto-Fluid Dynamics Division Report DOE/ER/03077-269 MF-107.

Lao L. L., Hirshman S. P. and Wieland R. M. (1981) Physics Fluids 24, 1431.

PAN L. J. and FU D. (1989) Plasma Phys. contr. Fusion 31, 993

Wu H. M. and PAN L. J. (1983) Acta Phys. Sin. 33, pp. 1100-1111. 